\title{
Temperature error of Hall-effect and magnetoresistive commercial magnetometers
}

\author{
Michal Nowicki ${ }^{1}$, MaCIEJ KACHNIARZ ${ }^{2}$, RoMAN SzEWCZYK ${ }^{1}$ \\ ${ }^{1}$ Institute of Metrology and Biomedical Engineering, Warsaw University of Technology \\ św. Andrzeja Boboli 8, 02-525 Warsaw, Poland \\ e-mail:m.nowicki@mchtr.pw.edu.pl \\ ${ }^{2}$ Industrial Research Institute for Automation and Measurements PIAP \\ al. Jerozolimskie 202, 02-486 Warsaw, Poland \\ e-mail:mkachniarz@piap.pl
}

(Received: 30.09.2016, revised: 17.02.2017)

\begin{abstract}
The paper presents a special measurement system for investigation of temperature influence on the indication of commercially available sensors of the magnetic field. Utilizing the developed system, several magnetoresistive and Hall-effect sensors were investigated within the temperature range from $-30^{\circ} \mathrm{C}$ to $70^{\circ} \mathrm{C}$. The obtained results indicate that sensitivity of most of the investigated sensors is unaffected, except the basic magnetoresistive device. However, Hall-effect sensors exhibit considerable temperature drift, regardless of the manufacturer.
\end{abstract}

Key words: magnetic field measurement, magnetometers, Hall-effect, magnetoresistive, temperature error

\section{Introduction}

An additional error induced by the influence of temperature is an important problem in almost all kinds of measurements performed in modern science and industry. The temperature influence is very harmful, especially in precise laboratory measurements. Presently developed measurement devices are often equipped with systems compensating influence of the temperature on the measurement results [1-3] or are designed in a way allowing self-temperature compensation $[4,5]$.

Also measurements of the magnetic field, widely utilized in many industrial processes, are influenced by the temperature. In this paper, the investigation of an additional, temperature induced error of various commercially available magnetometers is presented. Investigated magnetometers were based on both the magnetoresistive effect [6] and the Hall-effect [7]. Obtained results indicate that for some temperature range, investigated commercial magne- 
tometers are more or less resistant to the influence of the temperature. But for the significantly low or high temperatures, an additional error of the measurement is clearly observable. There is also a significant difference of an error occurrence mechanism between the various types of sensors.

\section{Measurement stand}

For this research, a special test stand was designed and built, consisting of precision Helmholtz coils used as a magnetic field standard, and $0.01^{\circ} \mathrm{C}$ precise PolyScience AD 07R-40-A12E Cryostat for temperature setting. The developed test stand schematic is presented in Fig. 1, the photograph of the actual setup is presented in Fig. 2.

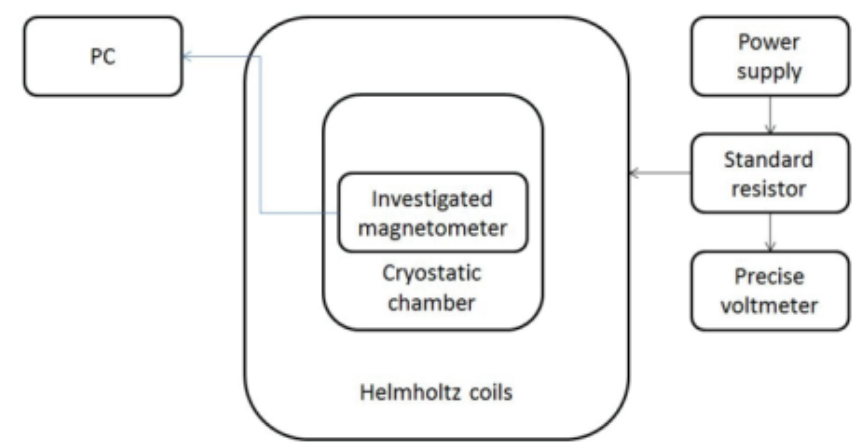

Fig. 1. Developed measurement stand for investigation the temperature influence on the magnetometers

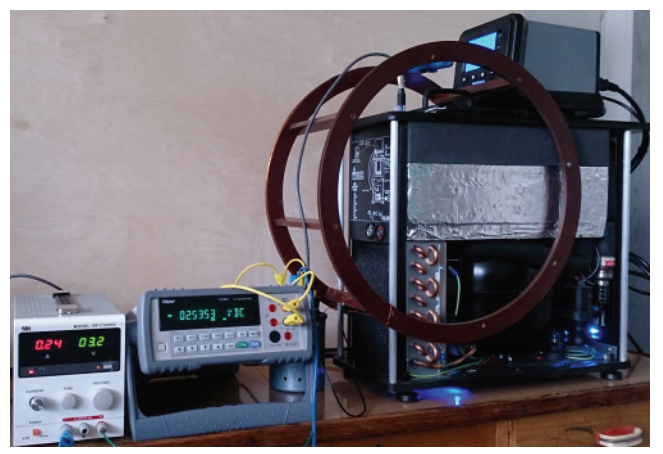

Fig. 2. Photo of the measurement stand

The Helmholtz coils were powered from a modified, precise laboratory power supply in a constant current mode. The current was measured with $0.01 \%$ precision on standard $1 \Omega$ resistor with Tonghui TH1961 voltmeter. The Earth's magnetic field component was compensated to within $\pm 0.1 \mu$ T range. 
The test stand allowed measuring the magnetometer's response in the $\pm 100 \mu \mathrm{T}$ magnetic field range, in $-30^{\circ} \mathrm{C}$ to $70^{\circ} \mathrm{C}$ temperature range, which is $\pm 50^{\circ} \mathrm{C}$ from the nominal $20^{\circ} \mathrm{C}$ operating temperature.

\section{Measurement results}

In the paper, the temperature dependence characteristics of four different magnetometers are presented. There were two triaxial magnetoresistive magnetometers (Honeywell HMR 2300 and HMC 5883L), one triaxial Hall-effect sensor (Metrolab THM 1176-LF) and one, one-axis Hall-effect sensor (Lakeshore 455 Gaussmeter with HMFT-3E03-VR probe) investigated. Based on the measurement results, linear fitting was performed, and temperature dependence of offset and sensitivity was determined. It was found that for most magnetometers, except the basic magnetoresistive one, the sensitivity was unaffected by the temperature in the investigated $20^{\circ} \mathrm{C} \pm 50^{\circ} \mathrm{C}$ range. On the other hand, the offset was negligible for the magnetoresistive sensors, but significant for the Hall-effect sensors, regardless of the manufacturer. In Fig. 3, there are measurement results of the Honeywell HMC 5883L. In Fig. 4 and Fig. 5, there are $\%$ change values of offset and sensitivity in relation to base $20^{\circ}$ values.

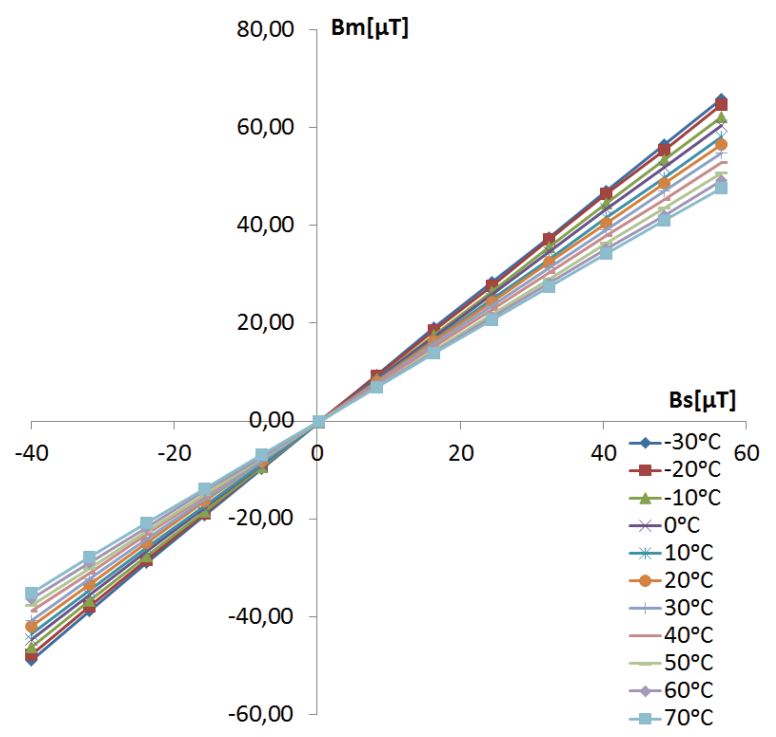

Fig. 3. Measurement results for magnetoresistive sensor Honeywell HMC 5883L. $B_{s}-$ magnetic field set by the Helmholtz coils, $B_{m}$ - magnetic field indicated by the sensor

There is a strong, highly linear correlation between the sensitivity of the device and the operating temperature. HMC 5883L is a very simple magnetoresistive sensor, which has no compensation circuit to reduce the influence of temperature on the operation of the device. On the other hand, the temperature influence on the offset is negligible. 


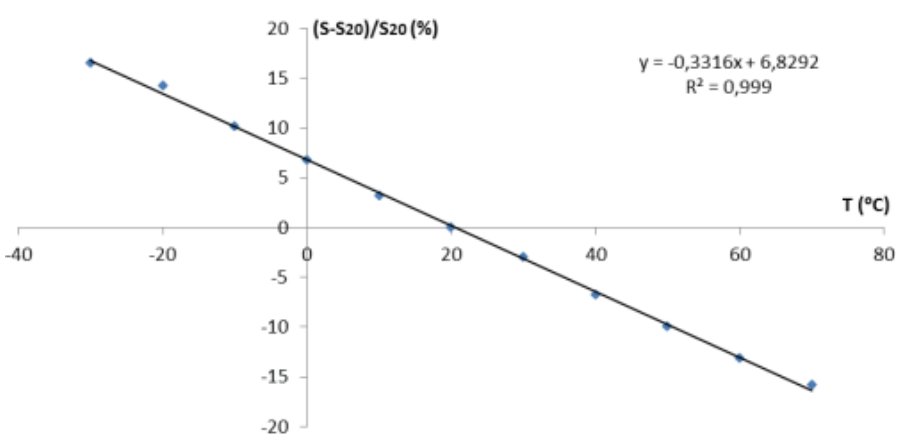

Fig. 4. Temperature dependence of sensitivity in relation to normal operating temperature $20^{\circ} \mathrm{C}$ for magnetoresistive sensor Honeywell HMC 5883L

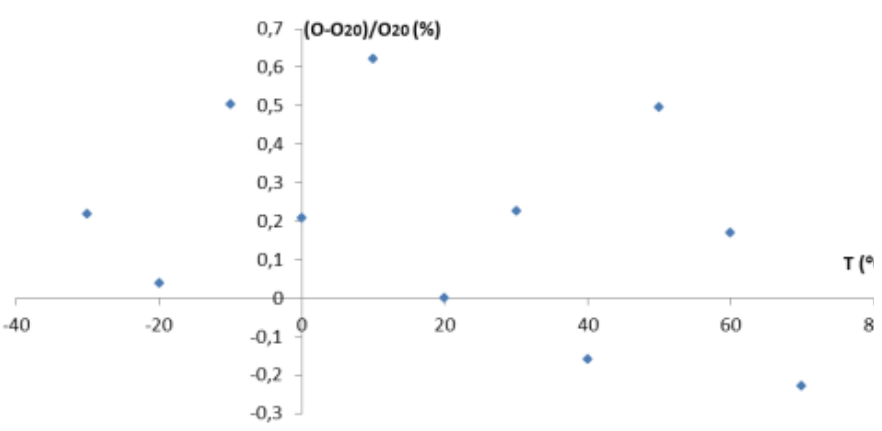

Fig. 5. Temperature dependence of offset value in relation to normal operating temperature $20^{\circ} \mathrm{C}$ for magnetoresistive sensor Honeywell HMC 5883L. The results indicate a negligible influence of temperature on offset values

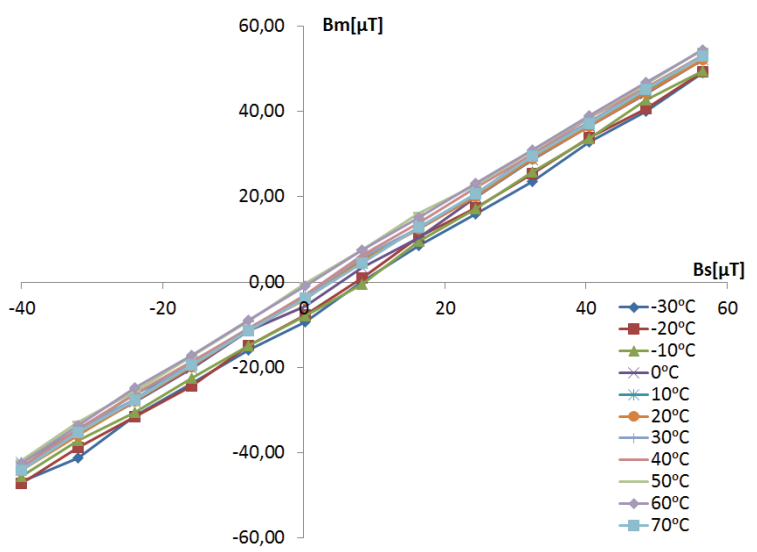

Fig. 6. Measurement results for Hall-effect Metrolab THM 1176-LF sensor. $B_{s}$ - magnetic field set by the Helmholtz coils, $B_{m}$ - magnetic field indicated by the sensor

In Fig. 6, the results for Metrolab THM 1176-LF are presented. In Fig. 7 and Fig. 8 there are $\%$ change values of offset and sensitivity in relation to base $20^{\circ}$ values of the sensor. 


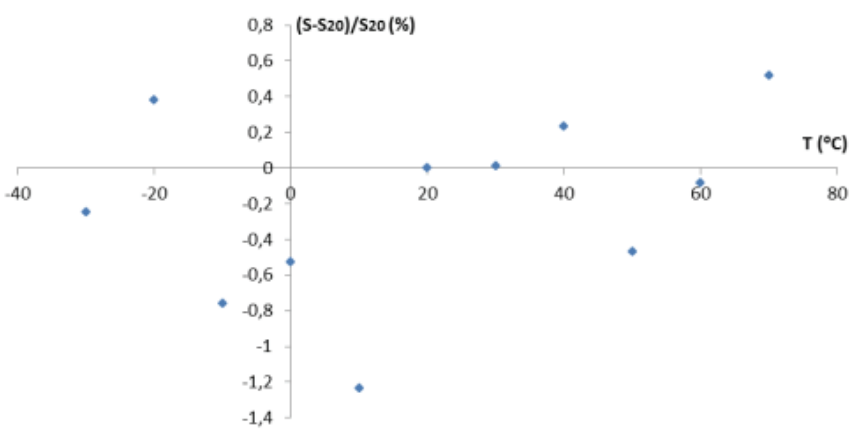

Fig. 7. Temperature dependence of sensitivity in relation to normal operating temperature $20^{\circ} \mathrm{C}$ for Halleffect Metrolab THM 1176-LF sensor. The results indicate negligible correlation of sensitivity and operating temperature

There is a significant, nearly linear correlation between the offset of the device and the operating temperature. On the other hand, the influence of the temperature on the sensitivity is negligible.

Very similar results were obtained for the Lakeshore 455 Gaussmeter with HMFT-3E03-VR probe. Again, the influence of the temperature on the offset was significant, while the influence of the temperature on sensitivity was negligible.

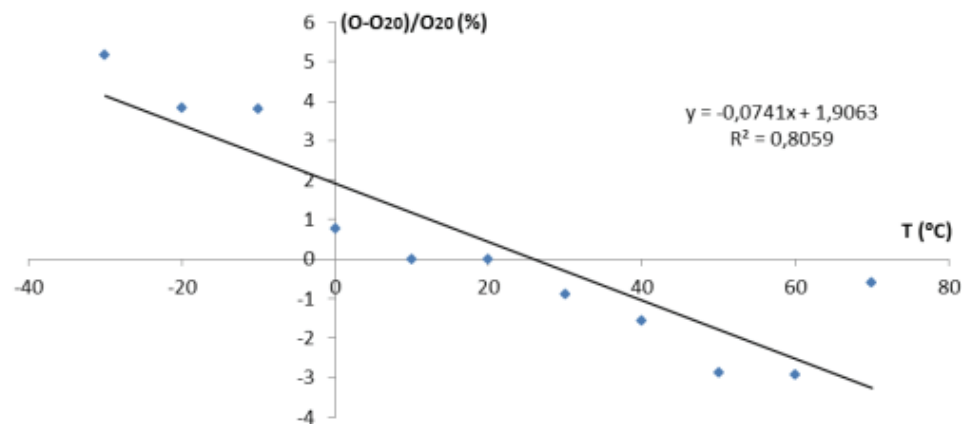

Fig. 8. Temperature dependence of offset value in relation to normal operating temperature $20^{\circ} \mathrm{C}$ for Hall-effect Metrolab THM 1176-LF sensor. The results indicate significant influence of temperature on offset values, with close to linear correlation

Table 1. Temperature dependence of sensitivity and offset in relation to normal operating temperature $20^{\circ} \mathrm{C}$ for investigated magnetometers

\begin{tabular}{c|c|c}
\hline Magnetometer type & $\begin{array}{c}\text { Temperature dependence } \\
\text { of sensitivity coefficient }\left(\mathbf{\%} /{ }^{\circ} \mathbf{C}\right)\end{array}$ & $\begin{array}{c}\text { Temperature dependence } \\
\text { of offset coefficient }\left(\% /{ }^{\circ} \mathbf{C}\right)\end{array}$ \\
\hline HMC 5883L & -0.33 & negligible \\
\hline HMR 2300 & negligible & negligible \\
\hline THM 1176-LF & negligible & -0.07 \\
\hline HMFT-3E03-VR & negligible & 0.25 \\
\hline
\end{tabular}


For the HMR 2300 triaxial magnetoresistive magnetometer, there was no observable influence of the temperature on offset and sensitivity in the $-30^{\circ} \mathrm{C}$ to $70^{\circ} \mathrm{C}$ range. Any temperature related error was lower than the uncertainty of the measurement stand and noise induced by the surrounding laboratory environment.

The results of experimentally measured temperature coefficients of offset and sensitivity for the investigated magnetometers are summarized in the Table 1.

\section{Conclusions}

Results of the performed investigation indicate that most of the commercially available magnetometers are not sensitive against temperature variations. However, in simple devices for less demanding applications, such as the popular HMC 5883L, strong dependence between functional parameters and temperature is observable. On the other hand, thanks to the highly linear temperature dependence of sensitivity, it can be easily compensated, as is the case in the HMR 2300.

Another interesting phenomena is the different mechanisms of temperature error appearance for magnetoresistive and Hall-effect sensors. For the magnetoresistive ones, the sensitivity is influenced and offset seemingly unaffected, while for the Hall-effect sensors the sensitivity is constant (or rather, precisely compensated), while the influence on offset is significant. It is evident even on comparison of measurement results in Fig. 3 and Fig. 6.

Thus, the developed test stand allows not only for measurement of temperature related error of the given magnetometer, but also for differentiation of the mechanism of error occurrence, and proper compensation method development.

\section{Acknowledgements}

This work was partially supported by statutory funds of Institute of Metrology and Biomedical Engineering, Warsaw University of Technology (Poland).

\section{References}

[1] Sosna Ch., Buchner R., Lang W., A Temperature Compensation Circuit for Thermal Flow Sensors Operated in Constant-Temperature-Difference Mode, IEEE Transactions on Instrumentation and Measurement, vol. 59, no. 6, pp. 1715-1721 (2010).

[2] Liu Z.J., Yu Y.S., Zhang X.Y., Chen C., Zhu C.C., Meng A.H., Jing S.M., Sun H.B., An Optical Microfiber Taper Magnetic Field Sensor with Temperature Compensation, IEEE Sensors Journal, vol. 15, no. 9, pp. 4853-4856 (2015).

[3] Juś A., Nowak P., Szewczyk R., Nowicki M., Winiarski W., Radzikowska W., Assessment of Temperature Coefficient of Extremely Stable Resistors for Industrial Applications, Advances in Intelligent Systems and Computing, vol. 317, pp. 297-306 (2015).

[4] Li Y., Wang J., Luo Z., Chen D., Chen J., A Resonant Pressure Microsensor Capable of SelfTemperature Compensation, Sensors, vol. 15, no. 5, pp. 10048-10058 (2015).

[5] Wang H., Feng Z., Ultrastable and highly sensitive eddy current displacement sensor using selftemperature compensation, Sensors and Actuators A: Physical, vol. 203, pp. 362-368 (2013).

[6] Tumański S., Thin film magnetoresistive sensors, CRC Press (2001).

[7] Ramsden E., Hall-Effect Sensors: Theory and Application, Newness (2006). 